\title{
Course Development Based on a Model for the Classification of Learning Progress
}

\author{
U. Maier and A. Löffler
}

\begin{abstract}
Examinations at the end of a one semester course at a University of Applied Sciences give at best a snap shot of the learning results at a given point of time. Unfortunately, this information is far from sufficient to analyze and evaluate the learning progress during the duration of a particular course. If one intends to continuously improve the course with respect to an optimization of the learning progress, a more detailed analysis is necessary. For this reason, a model has been developed based on the hypothesis that learning progress is mainly governed by two effects: accumulation and segregation. A comparison of predicted examination results with actual ones permits to identify - by means of an optimization process - two parameters representing the respective strengths (weights) of these effects. The values of these two parameters then allow to classify the learning progress within the respective course and to represent a basis for such a continuous course improvement process.
\end{abstract}

Index Terms-Accumulation effect, course development, learning progress, segregation effect.

\section{INTRODUCTION}

Due to the demographic development in Germany and the political boundary conditions, a rapid increase of student numbers on a mid-time range and also a shortage of qualified persons at work-force level are expected. For example, in the federal state Baden-Württemberg, the number of pupils leaving school with formal qualification for university steadily rose from 40,000 to about 70,000 within the last fifteen years. It is expected that this high level can be held for a few years before it continuously drops to about 54,000 until 2030 (information of the Statistical State Office of Baden-Württemberg). This poses a major challenge for Universities of Applied Sciences. With respect to these limiting factors the quality of teaching and the improvement of the learning progress are more and more important.

Especially, the drop-out rate as result of failed exams has to be decreased. At the study support center of Aalen University, we have developed several measures to support students in their first semesters [1].

Since the results of structural changes can only be seen with a delay of several years and since feedback loops can influence further development, system dynamic models seem to be an adequate tool to investigate complex systems arising in higher education. The application of the System Dynamics methodology began in the 1960s in the field of business

Manuscript received May 9, 2014; revised July 16, 2014. This work was financially supported by the German ministry of education and research (BMBF) under Grant 01PL11015.

The authors are with the Study Support Center, Aalen University, Aalen, CO $73430 \quad$ Germany (e-mail: Ulrike.Maier@htw-aalen.de, Axel.Loeffler@htw-aalen.de). administration [2]. Since then it has been applied to many fields including education [3]-[6]. For about two decades, the methodology is also utilized for questions arising in the education management domain.

In [7], [8] Kennedy gives a broad review of System Dynamics models for education policy issues. In [9], [10] Kennedy has researched on managing quality in higher education. He investigated System Dynamics models for school management and simulated the evolution of quality of teaching, student performance, staff costs, and total costs. From his results, he made a good point that higher education departments may, through the use of such a model, obtain useful insights into the likely impact of education policies on the attainment of quality related objectives.

In [11] a model and simulation of the teaching/learning process in a regular lesson of 45 minutes is presented, using system dynamics methods. The authors asked how a learner and a teacher really work and interact with each other. The time in their model is assumed to be 45 minutes, the length of a regular class lecture. They revealed the results that the advance knowledge about the types of teachers and learners warrants an efficient reengineering of the teaching-learning system.

In [12], the primary completion and the gross enrollment rate of education systems using a Systems Dynamics model for primary education, calibrated for the case of Nicaragua, is presented. The authors discuss the structure and the behavior of educational systems by considering the intake, repetition, drop-out, and graduation in primary school. Their model calculates the evolution of the stocks of students in primary school and is improvable to a model for capacity planning at universities.

We have introduced the idea of stocks and flows in [13] to describe the discrete time development of the stock of students in the semester at a University of Applied Sciences. We calculate the average number of students at each semester, the number of drop-out, and the number of graduation with two different admission scenarios. Our aim in this context is to optimize the enrolment sequence and predict future numbers of alumni, dropouts, and students in each semester.

In the present paper we introduce a system dynamics model which is used to simulate the learning progress regarding a standard semester at Universities of Applied Sciences of 15 weeks. Our model consists of two parts: accumulation and segregation effects. We first describe each effect separately and present simulation results showing the impact of corresponding characteristic parameters for each effect. Our first results for the segregation effect resemble representations showing Polya processes as described e.g. in [6], pp. 398. We combine the effects of accumulation and segregation in a combined model and apply this model to 
empirical data obtained from examination results of the study program "Industrial engineering" at Aalen University. We optimize the parameters of our model with respect to these data.

Since the detection of structural high-leverage parameters in our model is one of our goals at this point of our investigation, we have decided to formulate our model in form of mathematical difference and differential equations explicitly and run the simulation in Matlab/Simulink, instead of using one of the standard tools in system dynamics like STELLA, Powersim, Dynamo, Vensim, ithink or others. Thus, we are able to include or change particular parameters (perhaps nonvisible, but structurally important ones) that might be vital in the development of an adequate model for a certain system behavior. Especially, we hope to obtain a deeper insight into the learning process.

\section{BASIC INGREDIENTS OF THE MODEL}

\section{A. Empirical Data}

The empirical data inspiring the presented work are examination results of the years 2009-2011 from the study program of industrial engineering at Aalen University. Examination results at Aalen University are grouped into 5 grade levels.

- A: "very good" (school mark 1)

- B: "good" (school mark 2)

- C: "satisfactory" (school mark 3)

- D: "pass" (school mark 4)

- E: "fail" (school mark 5)

Since we need a closer look at a finer scale of the grades, we use a transformation between the grade levels and some performance levels representing finer marks. Table I explains the transformation explicitly. Three performance levels are merged to a grade level, where $\mathrm{A}+, \mathrm{E} 0$ and $\mathrm{E}$ - in practice are not really used. They are only defined for conformity reasons. In this article we use this transformation in both directions. As an example, the examination results in engineering physics during 5 consecutive semesters (a $1^{\text {st }}$ semester course, the lecturer of which is one of the authors) are depicted in Fig. 1 .

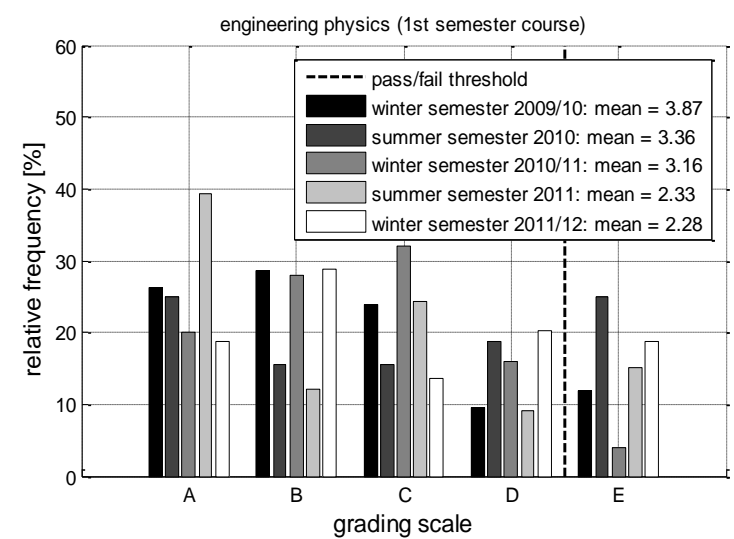

Fig. 1. Examination results in engineering physics (5 consecutive semesters).

In this particular case, the mean-values of the grades generally show an improving trend with a particularly high improvement from winter semester 2010/11 to summer semester 2011. Note that these data are the official results and are also available to the students.

TABLE I: TRANSFORMATION BETWEEN GRADE LEVELS AND PERFORMANCE

\begin{tabular}{c|c|c|c|c|c}
\hline \hline grade levels & A & B & C & D & E \\
\hline meaning & $\begin{array}{c}\text { very } \\
\text { good }\end{array}$ & good & satisfactory & pass & fail \\
\hline & $1(A+)$ & $4(B+)$ & $7(C+)$ & $10(\mathrm{D}+)$ & $13(\mathrm{E}+)$ \\
$\begin{array}{c}\text { performance } \\
\text { level }\end{array}$ & $2(\mathrm{~A} 0)$ & $5(\mathrm{~B} 0)$ & $8(\mathrm{C} 0)$ & $11(\mathrm{D} 0)$ & $14(\mathrm{E} 0)$ \\
& $3(\mathrm{~A}-)$ & $6(\mathrm{~B}-)$ & $9(\mathrm{C}-)$ & $12(\mathrm{D}-)$ & $15(\mathrm{E}-)$ \\
\hline \hline
\end{tabular}

Understanding the processes underlying the particular performances of the respective semesters is one of the motivations of the presented work. Identifying long-term trends with respect to a particular course and taking them into account for further improvement is another.

\section{B. Initialization for the Performance Levels}

For simulation purposes and to allow a more differentiated insight into the learning progress, we now use the 15 performance levels shown in Table I.

The basic hypothesis is that the initial state is proportional to normally distributed weights:

$$
L(n) \sim e^{-\frac{(n-m)^{2}}{s^{2}}}
$$

where

$n$ : number of performance level $(n=1,2,3, \ldots, 15)$

$L(n)$ : number of students in performance Level $n$

$m$ : mean value, e.g. 13

$s:$ standard deviation, e.g. 2

Remarks: The distribution of the students to performance levels as defined in (1) and depicted in Fig. 2 is used for all subsequent simulations as initial distribution. The performance levels have to be read from right to left, i.e. a lower performance level means improved knowledge and better grades. The mean value at 13 is chosen such that at least half of the group would have failed at the beginning. The threshold for failing lies between the grades D and E, i.e. at 12.5 (see the light gray dashed line in Fig. 2). Since the performance levels are discrete and finite, the distribution is first normalized and then rescaled to contain $s L$ students. In our simulations, we first use $s L=50$.

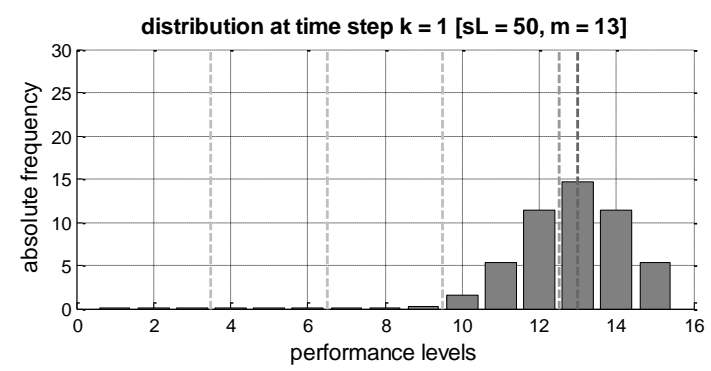

Fig. 2. Initial student distribution to performance levels.

Note that the typical course at Aalen University is a 4 hours-per-week course with a typical duration of 15 weeks at the end of which an examination takes place. Therefore, the chosen time step for the simulation model is 1 week, starting 
at week 1 with the initial distribution. During simulation, our initial distribution changes its shape according to the learning progress and moves to the left, i.e. to lower performance levels.

\section{The Basic Dynamic Hypothesis}

The basic hypothesis is that the dynamics of the learning progress is governed by two effects that are called "accumulation effect" and "segregation effect" in the context of this paper.

"Accumulation" describes the effect that it becomes more and more difficult to mentally incorporate new topics during the course (within the framework of finite course duration). This is assumed to be a kind of "negative scaling effect", since - with progressing time - it becomes more and more difficult

- To remember and recall all of the already learned topics

- To connect and link new topics correctly with already learned ones.

"Segregation" describes the (relative) vicious circle effect ("success to the successful"): students who have an above average knowledge at their disposal at a given moment of time

- Are (relatively) more able to successfully incorporate and connect new topics

- Are (relatively) more motivated to learn, because they already "feel" successful and want this emotion to last.

Both, the accumulation and the segregation effect, are subsequently modeled by means of transition probabilities. Note that "transition probabilities with polarities" are used in the context of this paper, i.e. a "positive" transition probability associated with a performance level $n$ indicates that there is a probability for the corresponding group of students to advance to the next lower (better) performance level. A "negative" transition probability on the contrary indicates that there is a probability to degrade to the next higher (poorer) performance level:

$$
\begin{aligned}
& p(n)>0 \Rightarrow n \rightarrow n-1 \\
& p(n)<0 \Rightarrow n \rightarrow n+1
\end{aligned}
$$

\section{MODEL DESCRIPTION}

\section{A. Accumulation Effect}

The accumulation effect is described by a probability distribution $p_{a c c}(n)$ :

$$
p_{a c c}(n)=1-e^{-\frac{n-1}{n_{0}}}
$$

with

$n$ : number of performance level $(n=1,2,3, \ldots, 15)$

$n_{0}$ : characteristic parameter of the accumulation effect (the higher $n_{0}$, the slower is the learning progress)

The transition probability of the $1^{\text {st }}$ performance level is set to $0: p_{a c c}(1)=0$. This means that if a student has reached the $1^{\text {st }}$ performance level, there is no probability to advance further within the framework of the course. The student already knows all that he is required to know.
Note that this kind of transition probability function is the solution of the following first order, time-continuous ordinary differential equation describing an exponentially saturating process, which is evaluated at discrete points (the performance levels):

$$
\dot{p}_{a c c}(t)=\frac{1-p_{a c c}(t)}{n_{0}} ; p_{a c c}(1)=0
$$

The update of the number of students at the performance levels $n$ is governed by the following difference equation:

$$
\begin{aligned}
L_{a c c}(n, k)=L_{a c c}(n, k-1) & +p_{a c c}(n+1) \cdot L_{a c c}(n+1, k-1) \\
& -p_{a c c}(n) \cdot L_{a c c}(n, k-1)
\end{aligned}
$$

where

$L_{a c c}(n, k)$ : number of students in performance level $n$ at week $k$

$p_{a c c}(n)$ : transition probability of the group at level $n$

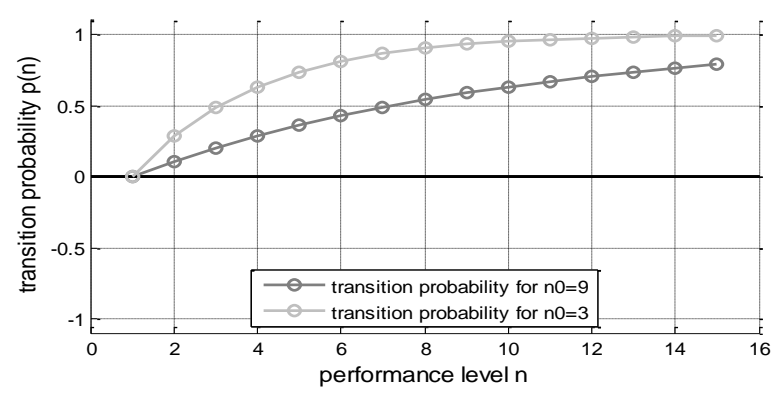

Fig. 3. Transition probabilities for $n_{0}=9$ and $n_{0}=3$.

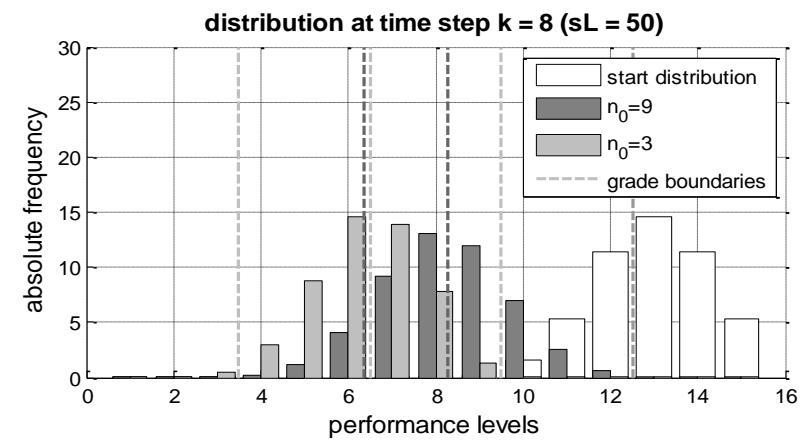

Fig. 4. Distribution of students to performance levels at week $k=8$ for $n_{0}=9$ and $n_{0}=3$.

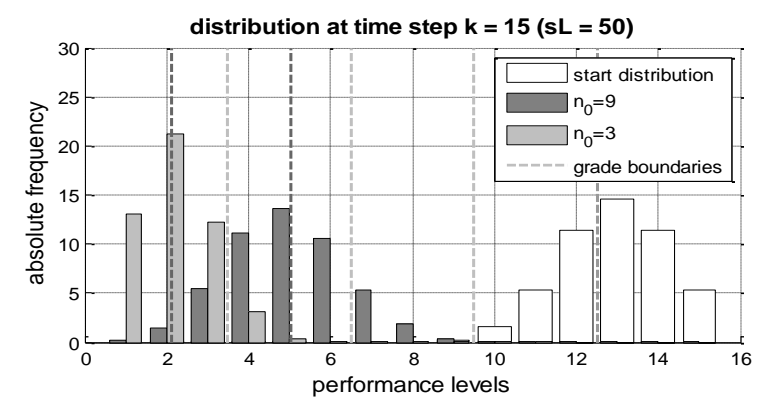

Fig. 5. Distribution of students to performance levels at week $k=15$ for $n_{0}=9$ and $n_{0}=3$.

Fig. 3 shows the transition probabilities for the characteristic parameter $n_{0}$ for $n_{0}=9$ and $n_{0}=3$ respectively. 
Fig. 4 and Fig. 5 present corresponding simulation results for these parameter values of $n_{0}$. Fig. 4 shows the result at time step $k=8$, i.e. week 8 , whereas Fig. 5 shows the result at week 15 , the last week of the semester.

It can clearly be seen that for $n_{0}=9$ there is a slower progression of learning than in the case of $n_{0}=3$, where a considerable part of the students reached the first three performance levels (obtaining an A in the subsequent exam), see Fig. 5. This is due to the different transition probability functions visible in Fig. 3.

The graphs have to be read from right to left (see Remarks at the end of section B). The longer the course lasts, the more difficult it is to transfer to a better (lower) performance level, i.e. the transition probability is becoming smaller. The accumulation effect implicitly involves a process of oblivion. As a secondary effect, note the slight dispersion (increasing standard deviation) as the mean-value progresses.

\section{B. Segregation Effect}

The segregation effect is described by a probability distribution $p_{\text {seg }}(n, k)$ of the group at the level $n$ in week $k$ :

$$
p_{\text {seg }}(n, k)=p_{0} \cdot \frac{\bar{n}(k)-n}{N}
$$

where

$N$ : total number of performance levels (here: $N=15$ )

$n$ : number of actual performance level $(n=1,2,3, \ldots, \mathrm{N})$

$p_{0}$ : characteristic parameter of the segregation effect (the higher $p_{0}$, the faster is the learning progress)

$\bar{n}(k)$ : average performance level at the $\mathrm{k}^{\text {th }}$ time-step

where $\bar{n}(k)$ is calculated by

$$
\bar{n}(k)=\frac{\sum_{n=1}^{N} L_{s e g}(n, k) \cdot n}{\sum_{n=1}^{N} L_{s e g}(n, k)}
$$

$L_{\text {seg }}(n, k)$ : number of students in performance level $n$ week $k$

Note that - in contrast to the transition probability calculation for the accumulation effect - the transition probabilities $p_{\text {seg }}(n, k)$ do not only depend on the performance level $n$ itself, but also on the time-step $k$. Moreover, the transition probabilities are not all positive (positive are only those performance levels "above" average, i.e. $n<\bar{n}(k)$ ), but may also be negative (for those performance levels "below" average, i.e. $n>\bar{n}(k))$. Finally, if the absolute value of a transition probability exceeds 1 , the transition probability is saturated at 1 or -1 , respectively.

The update of the number of students at the performance levels $n$ is governed by the following difference equation:

$$
\begin{aligned}
L_{s e g}(n, k) & =L_{s e g}(n, k-1)-\left|p_{\text {seg }}(n, k)\right| \cdot L_{\text {seg }}(n, k-1) \\
& +p_{\text {seg }}(n+1, k) \cdot L_{\text {seg }}(n+1, k-1) \quad\left[\text { if } p_{\text {seg }}(n+1, k)>0\right] \\
& -p_{\text {seg }}(n-1, k) \cdot L_{\text {seg }}(n-1, k-1) \quad\left[\text { if } p_{\text {seg }}(n-1, k)<0\right]
\end{aligned}
$$

As initial distribution for the number of students $L$ we take the one defined in (1) (see Fig. 2). Fig. 6 gives the transition probabilities for two values of the parameter $p_{0}$ : $p_{0}=3 / 15=0.2$ and $p_{0}=1 / 15=0.067$. Fig. 7 and Fig. 8 show the learning progression at week 8 and week 15 for these values of the parameter $p_{0}$.

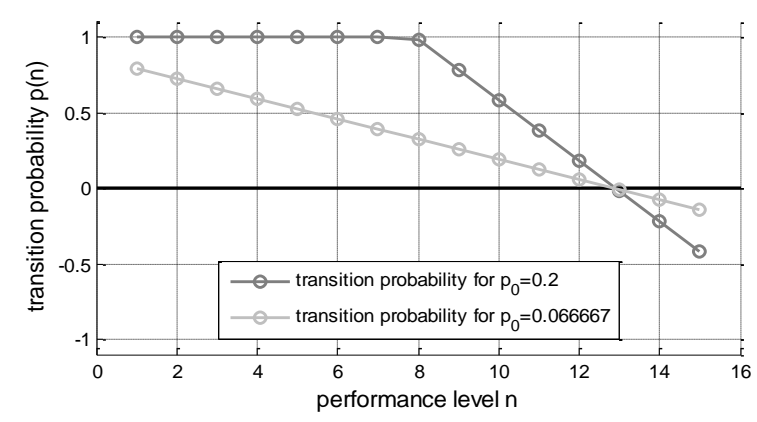

Fig. 6. Transition probabilities for $p_{0}=0.2$ and ${ }_{p_{0}}=0.067$

It can clearly be seen that there is faster progression of learning and also a faster "drifting apart" from the performance levels for $p_{0}=0.2$ than in the case of $p_{0}=0.067$. This can be explained by the different transition probability functions visible in Fig. 6, which are now also dependent on $k$. It can be seen that there are some students who deteriorate, which can be seen in Fig. 6 from negative values of the transition probability. Once students have reached performance level 12, they improve further. For segregation parameter $p_{0}=0.2$ the improvement takes place even with probability 1 as soon as students have reached performance level 8 .

As a secondary effect, the standard deviation is steadily increasing as one could expect as the effect of a segregation process.

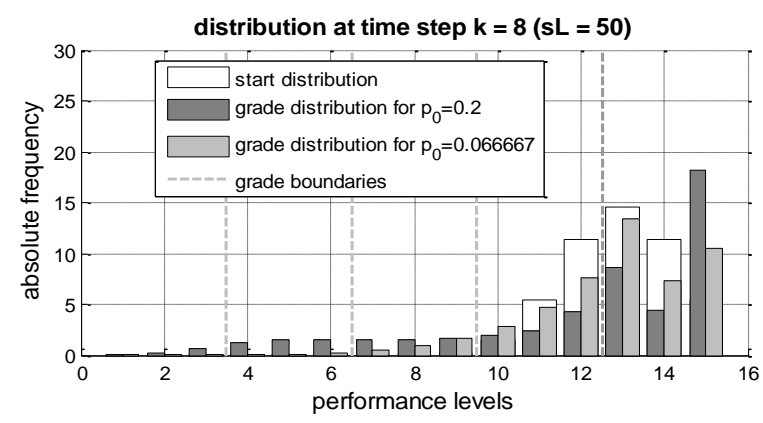

Fig. 7. Distribution of students to performance levels at week $k=8$ for $p_{0}=0.2$ and $p_{0}=0.067$.

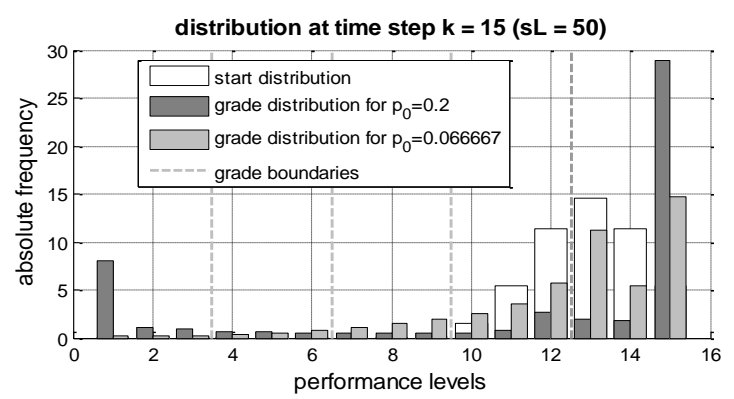

Fig. 8. Distribution of students to performance levels at week $k=8$ for $p_{0}=0.2$ and $p_{0}=0.067$. 


\section{Dynamics of a Combined Model}

As has been shown in the sections $A$ and $B$, the parameter $n_{0}$ of the accumulation effect and the parameter $p_{0}$ of the segregation effect have an important influence on the described effects and their speed. We are now interested to combine the two effects.

In a combined model, this combination is achieved by adding the two transition probability functions (see (2) and (5)) and by an addition of the update formulae for the number of students at the performance levels (see (4) and (7)):

$$
\begin{gathered}
p(n, k)=p_{a c c}(n)+p_{s e g}(n, k) \\
L(n, k)=L_{a c c}(n, k)+L_{s e g}(n, k)
\end{gathered}
$$

To simulate empirical data as good as possible we then have to identify the two characteristic parameters $n_{0}, p_{0}$ in a combined model, such that the simulated performance levels "fit" to the empirical data. As data we have chosen examination results for several courses (see Chapter IV).

\section{Optimization Process}

To identify the characteristic parameters we perform an optimization process. Before one can start the optimization process, two steps have to be carried out:

- first, the 15 simulated performance levels have to be aggregated to the 5 grades (see Table I).

- second, a distance measure between the simulated and the actual grades has to be defined:

$$
d\left(\vec{x}_{1}, \vec{x}_{2}\right)=100 \cdot \sum_{i=1}^{5}\left|x_{1 i}-x_{2 i}\right|
$$

where

$\vec{x}_{1}, \vec{x}_{2}$ : normalized distributions (i.e. $\sum x_{1 i}=\sum x_{2 i}=1$ ) of grade levels

$i:$ index $(i=1 . .5:$ A to $\mathrm{E})$

In our model we choose the vector $L$ constructed as in (9) for $\vec{x}_{1}$ where three components of $L$ are merged to build each of the 5 component of the vector $\vec{x}_{1}$. The vector $\vec{x}_{2}$, correspondingly, is constructed from the grade distribution of the examination results. Note that the grade level distributions have to be normalized to obtain a distribution independent from the total number of students in one semester. The factor 100 in (10) allows an interpretation of the result as a percentage. The "distance" $d$ between two grade level distributions is always greater than or equal to $0 \%$ (but could become greater than 100\%).

The distance measure $d$ has to be minimized with respect to the parameters $n_{0}$ and $p_{0}$. We divided the $n_{0}, p_{0}$-plane into parts and evaluated the distance measure $d$ at the corresponding grid points thus finding the minimal distance. The next step will be to apply a typical optimization algorithm to ensure that errors between simulation and real data are independent of the optimization algorithm used.

We will illustrate the results for two examples in the next chapter: The examination results in engineering mathematics and engineering mechanics for the winter semester 2011/12.

\section{ApplicAtion of THE COMBined Model}

\section{A. Engineering Mathematics Examination}

We apply our model to the data set from the $2^{\text {nd }}$ semester engineering mathematics course in industrial engineering at Aalen University (winter semester 2011/12):

- A-grade: 4 students

- B-grade: 7 students

- C-grade: 6 students

- D-grade: 11 students

- E-grade: 12 students

In Fig. 9, the distance is graphically depicted as a function of $n_{0}, p_{0}$. Although, the surface appears to be quite rough, there clearly is a global minimum $d^{o p t}=10 \%$ of the distance measure $d$ in (10) for the parameters $n_{0}^{o p t}=37.5, p_{0}^{o p t}=0.0933$.

The roughness is typical for statistical processes (Brownian motion, Martingal processes) and is even worse for a finer division of the $n_{0}, p_{0}$-plane.

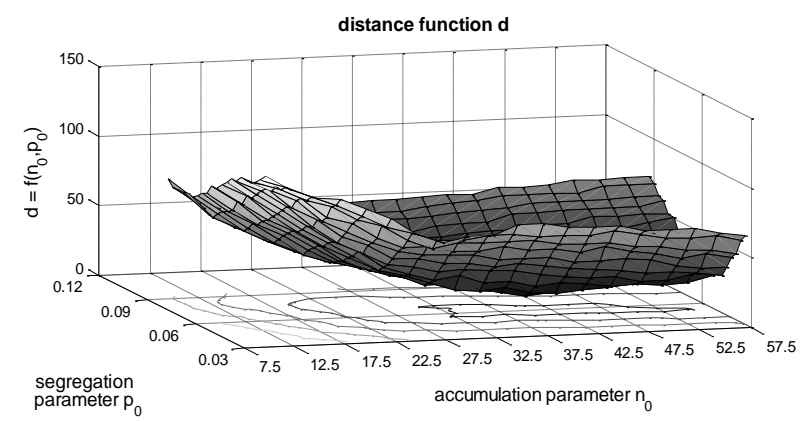

Fig. 9. Surface plot (with contour lines below) of the distance function for the engineering mathematics course in the $n_{0}, p_{0}$-plane.

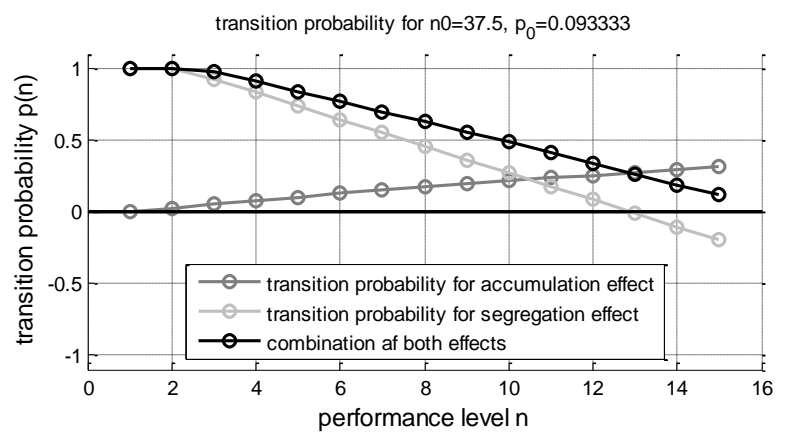

Fig. 10. Transition probabilities for the optimal parameters $n_{0}, p_{0}$ in the combined model at time step $k=15$.

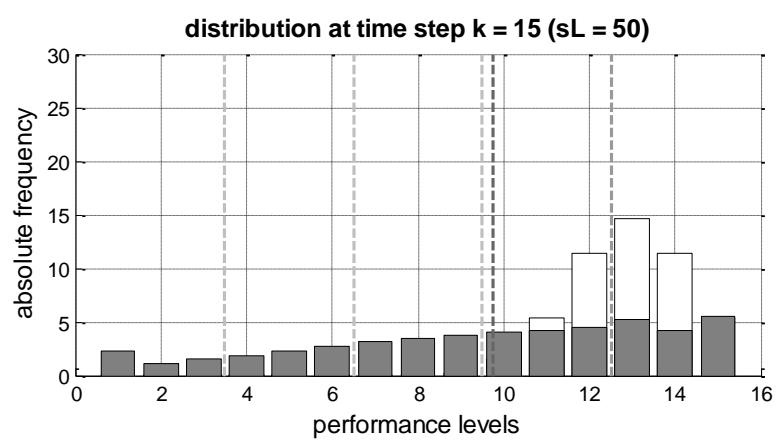

Fig. 11. Distribution of the performance levels for the optimal parameters $n_{0}, p_{0}$ in the combined model at week $k=15$. 


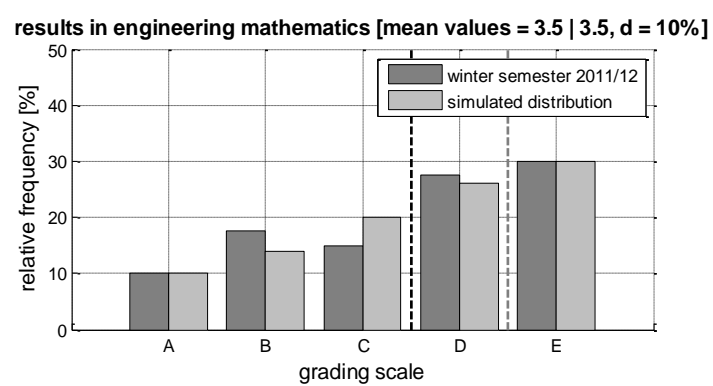

Fig. 12. Comparison of the simulated distribution of grade levels for the optimal parameters $n_{0}, p_{0}$ with the actual one (at week $k=15$ ).

Fig. 10 presents the transition probabilities for the accumulation effect with $n_{0}^{o p t}=37.5$, for the segregation effect with $p_{0}^{\text {opt }}=0.0933$ and for the combination of the effects at time step $k=15$. Fig. 11 shows the corresponding distribution of the performance levels for the combined model at week $k=$ 15 (the last week of the semester).

Fig. 12, eventually, presents the comparison of the simulated learning progress (light gray) with the empirical examination results in engineering mathematics (dark gray).

One can interpret the result as a learning process dominated by a very slow accumulation process $\left(n_{0}^{\text {opt }}=37.5\right)$ overlaid by a moderate segregation process $\left(p_{0}^{o p t}=0.0933\right)$.

There is a good agreement for grade $\mathrm{A}$ and $\mathrm{E}$, but at the grades $\mathrm{B}$ to $\mathrm{D}$ we feel that the agreement maybe could be improved. The final distance of $10 \%$ represents a good comparability, but also indicates that further processes, apart from accumulation and segregation, may be involved.

\section{B. Engineering Mechanics Examination}

We now apply our model to a data set from a $1^{\text {st }}$ semester engineering mechanics course in industrial engineering at Aalen University (winter semester 2011/12):

- A-grade: 26 students

- B-grade: 12 students

- C-grade: 10 students

- D-grade: 9 students

- E-grade: 4 students

In Fig. 13, the distance is graphically shown as a function of $n_{0}, p_{0}$, again. The surface is very rough again.

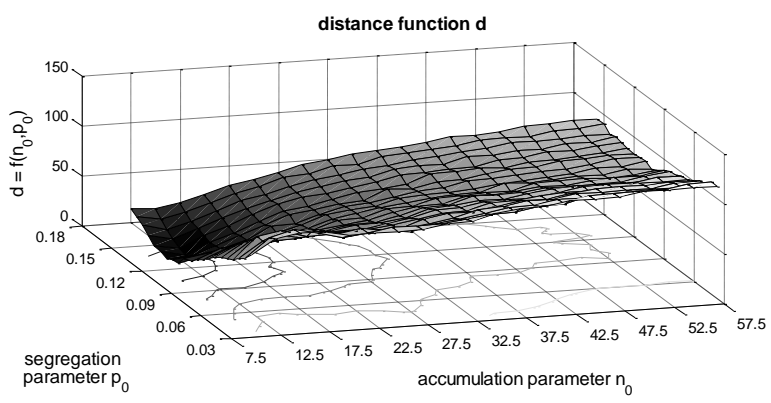

Fig. 13. Surface plot (with contour lines below) of the distance function for the engineering mechanics course in the $n_{0}, p_{0}$-plane.

The distance measure $d$ for the engineering mechanics course looks completely different from the distance function of the engineering mathematics course. The minimum of the distance function $d$ in (10) in this case can be found to be $d=$
$8 \%$ for the parameters $n_{0}^{o p t}=10.0, p_{0}^{o p t}=0.133$.

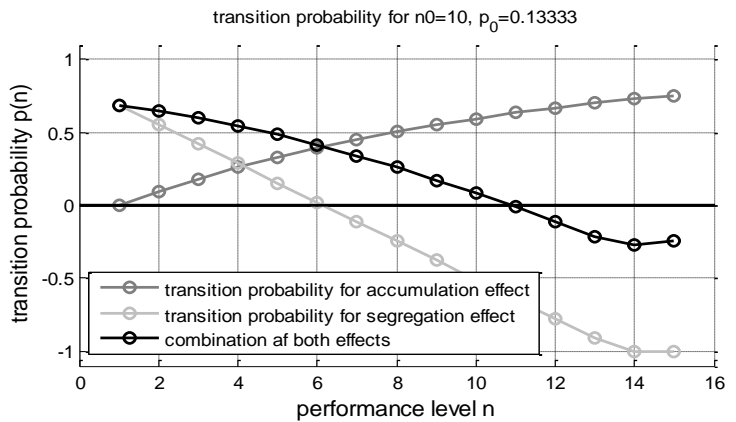

Fig. 14. Transition probabilities for the optimal parameters $n_{0}, p_{0}$ in the combined model at time step $k=15$.

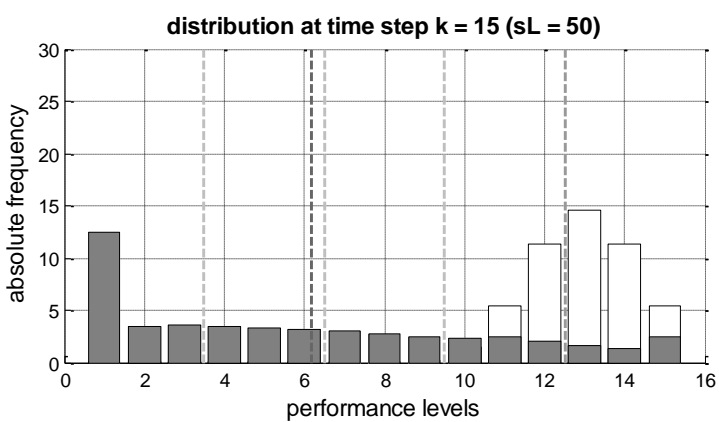

Fig. 15. Distribution of the performance levels for the optimal parameters $n_{0}, p_{0}$ in the combined model at week $k=15$.

Fig. 14 presents the transition probabilities for the accumulation effect, for the segregation effect and for the combination of both effects with the optimal parameters at time step $k$ $=15$. Fig. 15 shows the corresponding distribution of the performance levels for the combined model at week $k=15$ (the last week of the semester), again. The simulation in Fig. 16 shows a good agreement with the empirical data at grade $\mathrm{B}$ and $\mathrm{C}$. At the other grades an improvement could be better, again.

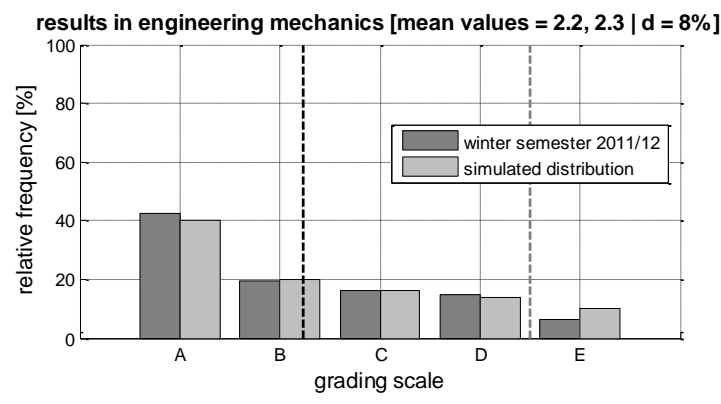

Fig. 16. Comparison of the simulated distribution of grade levels for the optimal parameters $n_{0}, p_{0}$ with the actual one at week $k=15$.

The obvious difference in the actual grade scales and the mean of the two courses $\left(2^{\text {nd }}\right.$ semester engineering mathematics and $1^{\text {st }}$ semester engineering mechanics) may stem from various reasons:

- the intrinsic complexity of the mathematics course $\left(2^{\text {nd }}\right.$ semester) is much higher than that of the mechanics course $\left(1^{\text {st }}\right.$ semester $)$

- the transfer part (new problems) of the mathematics examination is higher than that of the mechanics examinations 
- in mechanics more additional tuition (e.g. exercise lessons held by students of higher semesters) had been available

- more previous examinations (with solutions) had been available for mechanics than for mathematics

- the $1^{\text {st }}$ semester had a better respective initial performance level distribution than the $2^{\text {nd }}$ semester (the school grades of the first semester students were in average significantly better than those of the $2^{\text {nd }}$ semester students)

Further investigations may help to identify the most dominant reason(s).

\section{CONCLUSION}

Starting from the dynamic hypothesis that the learning process of a course at university level is mainly governed by two effects, the accumulation effect (negative scaling effect) and the segregation effect ("success to the successful"), a time-discrete transition probability model comprising these two effects has been developed.

Due to the lack of data concerning the pre-knowledge of a standard group of students at the beginning of most courses, we started our investigations with an arbitrary initial normal distribution of performance levels. The simulation results show the development of a "standard" course governed by accumulation and segregation, respectively, and illustrate the influence of the characteristic parameters.

Empirical data have been available from the courses of engineering physics $\left(1^{\text {st }}\right.$ semester), engineering mechanics $\left(1^{\text {st }}\right.$ semester) and (advanced) engineering mathematics $\left(2^{\text {nd }}\right.$ semester) over the last 2 to 3 years within the framework of the industrial engineering study program (B.Eng.) at Aalen University. Exemplarily, an optimization process has been carried out to fit the simulated results to the empirical data. This process yielded values for the characteristic parameters $n_{0}$ (accumulation parameter) and $p_{0}$ (segregation parameter).

By means of this optimization process and the values for the characteristic parameters obtained in this way, the learning processes of two separate courses could be analyzed and compared.

To our minds, the following points represent interesting aspects for future work in this area:

- Measurements of the initial state and measurements of intermediate states are vital to validate/reject the normal distribution of initial performance levels hypothesis. Since at Aalen University we offer introductory mathematics courses for student beginners (with tests checking the knowledge of the students at different time steps) we meanwhile have a more realistic initial distribution of the student numbers at performance levels available. To exploit this distribution, will be one of our next steps.

- The analysis of the development of the intermediate distributions during the course, e.g. in terms of mean-values and standard deviations, could hold valuable insights.

- The optimization process, being a very basic one at the moment, has to be improved as well as to be refined.

- The influence of improvement measures (e.g. reduction/restructuring of course contents, additional tuition [exercise lessons, question hours, examination preparation courses], etc.) has to be included into the simulation model and compared to reality.

- Further effects on the learning progress, apart from accumulation and segregation, might be taken into account.

- The two sub-models have to be analyzed further with respect to stability, steady-states and "conservation of students".

- The comparison with a time-continuous version of the model (e.g. a Vensim implementation) could be beneficial in terms of comparison of simulation results as well as communicability.

- The comparison to other modeling approaches ("physics of socio-economic systems") also represents an interesting aspect.

- The development of future courses should be observed in the framework of the presented model. In particular, an analysis of the time-series of the model's main parameters $\left(\mathrm{n}_{0}, \mathrm{p}_{0}\right)$ could yield information about long-term trends.

- The transfer of the results to other areas, e.g. staff development in the framework of organization design, could also be beneficial.

We simulated the "natural" (continuous) learning process, not taking into account the peaks in the learning effort short time before the examination. In a pilot test we tried to enforce the continuous learning process by weekly tests. The results and a simulation of the learning progress in this context are planned for another publication.

\section{APPENDIX}

We give a short survey of programming code segments in MATLAB showing how the calculation of the performance levels is realized. The dots ... indicate further lines of code not necessary to understand the main algorithms. Update procedure of the accumulation performance levels:

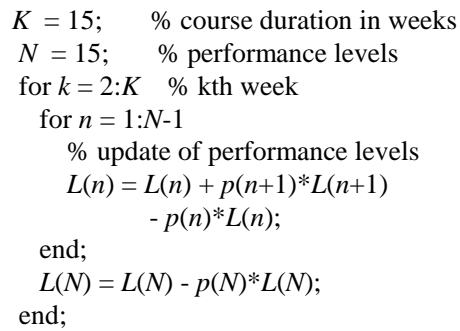

Update procedure of the segregation performance levels:

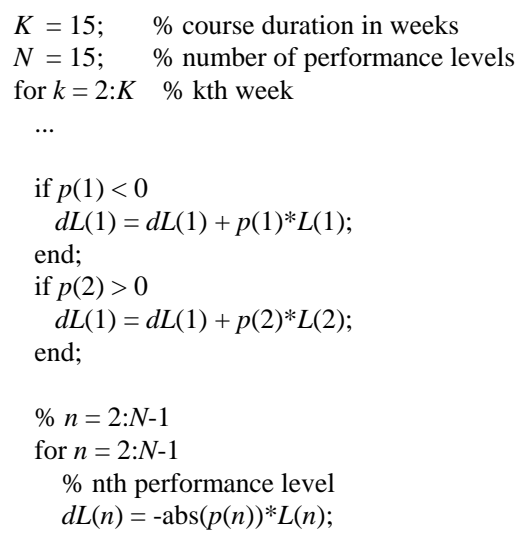




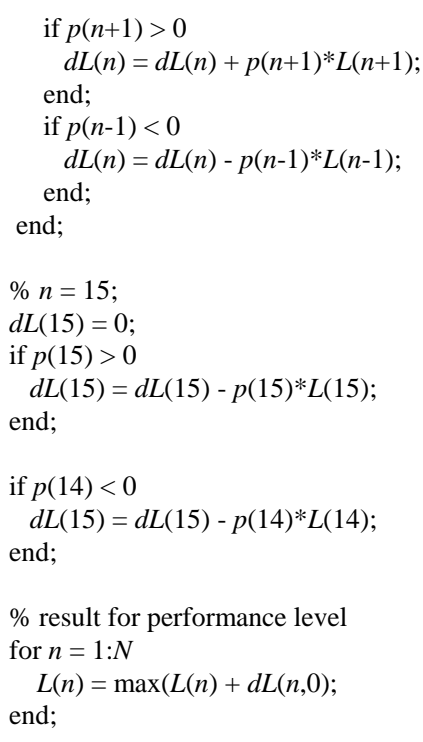

For the combined model we combined the procedures.

\section{REFERENCES}

[1] V. Nagengast, M. Hommel, and A. Löffler, "First phase of studies at the University of applied sciences Aalen - professional support and analysis of deficiencies," in Proc. $1^{\text {st }}$ HDMINT Symposium 2013, pp. 200-209, Nürnberg, Germany, 2013.

[2] J. W. Forrester, Urban Dynamics, Pegasus Communications, 1969.

[3] J. Gharajedaghi, System Thinking - Managing Chaos and Complexity, Elsevier, Inc., 2011.

[4] J. Morecroft, Strategic Modelling and Business Dynamics- A Feedback Systems Approach, John Wiley and Sons Ltd, 2007 (reprinted 2011).

[5] H. Nuhoglu and M. Nuhoglu, "System dynamics approach in science and technology education," J. Turkish Science Education, vol. 4, no. 2 , September 2007.

[6] J. D. Sterman, Business Dynamics - Systems Thinking and Modeling for a Complex World, McGraw-Hill Higher Education, 2000.

[7] M. Kennedy, "A taxonomy of system dynamics models of educational policy issues," in Proc. 29th System Dynamics Conf., Washington DC, US 2011.

[8] M. Kennedy, "A review of system dynamics models of educational policy issues," in Proc. 29th International Conf. of the System Dynamics Society, 2011.

[9] M. Kennedy, "Some issues in system dynamics model building to support quality monitoring in higher education," in Proc. 16th System Dynamics Conf., Quebec City, Canada, 1998.
[10] M. Kennedy, "A pilot system dynamics model to capture and monitor quality issues in higher education institutions experiences gained," in Proc. 16th System Dynamics Conf., Quebec City, Canada, 1998.

[11] N. Eftekhar and D. R. Strong, "Towards dynamic modeling of a teaching / learning process part 3: The simulation model," Int. J. Enging Ed. vol. 15, no. 3, pp. 169-190, 1999.

[12] P. Guevara, L. Lopez, and R. Zuniga, "Forecasting primary education efficiency," in Proc. System Dynamics Conference, 2005.

[13] Y. Endres, A. Löffler, and U. Maier, "A system dynamics model for the admission management of study programs," in Proc. Konferenz für Wirtschafts- und Sozialkybernetik KyWi 2013, pp. 333-346, Verlag Duncker and Humblot, Berlin, 2013.

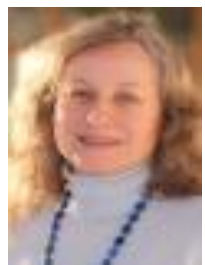

Ulrike Maier was born in Duisburg, Germany, 1963 She did studies in mathematics at the University Dortmund, Germany. Then she got her university diploma in mathematics 1990, and $\mathrm{PhD}$ in mathematics from the University Dortmund, Germany, 1994.

She is an assistant at Chairs in Applied Mathematics in the Universities Dortmund and Giessen, Germany, from 1990 to 2002 . Her research is in the fields of medical technical solutions and automotive production at the Fraunhofer Institute for "algorithms and scientific computing" (SCAI) at Sankt Augustin, Germany, from 2002 to 2004. She did research for Zeiss SMT AG, Oberkochen, Germany, from 2005 to 2007. She was an assistant at the Chair of Optoelectronics in the University Mannheim, Germany, from 2007 to 2008. She was a lecturer at the University of applied sciences at Heidenheim, Germany, spring 2008. She was an assistant at the KIZ of the University Ulm, Germany, from 2008 to 2011. Since 2012, she has been an assistant at the study support center of the University of applied sciences Aalen, Germany. Her current research interests are in approximation theory, scientific computing, and learning processes.

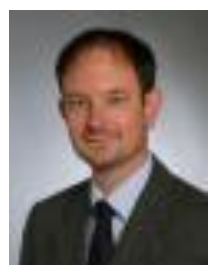

Axel Löffler was born in Karlsruhe, Germany, 1969 He did his studies in physics at the Universities of Karlsruhe, Germany, and Grenoble, France. The he got his university diploma (master level) in physics from the University of Karlsruhe in 1996, and $\mathrm{PhD}$ degree in electrical engineering from the University of Paderborn in 2000. He was a systems engineer in satellite technology at EADS-Astrium, Friedrichshafen, Germany, from 2000 to 2003, and a development engineer in the field of internal combustion engines at Bosch, Stuttgart, Germany, from 2004 to 2009. Since 2009 he has been a professor for mathematics, physics and system dynamics at the University of Applied Sciences at Aalen, Germany. He has been the head of the study support center since 2011. His research interests are in the application of system dynamics to various fields, in particular in the areas of renewable energies and learning processes.

Prof. Dr. Löffler is a member of the institute of applied system dynamics (IAS), System dynamics society, Deutsche Gesellschaft für Systemdynamik, Verein Deutscher Ingenieure (VDI), Deutsche Physikalische Gesellschaft (DPG), Hochschul Lehrer bund (Hlb). 